Okajimas Fol. anat. jap., 50 : 91-106, 1973.

\title{
Terminations of the Primary Trigeminal Fibers in the Rat, in Relation with Sensory Cortical Fibers
}

\author{
By
Shozo Matano, Tôru Hiura, Yoshio Shigenaga and Akira Sakai \\ Department of Anatomy, Osaka University Dental School, \\ Osaka, Japan \\ -Received for Publication, September 12, 1972-
}

\section{Introduction}

Many researchers have reported on the terminations of the trigeminal nerve in various animals including man. The recent experiments were especially studied on the nerve terminals based upon the individual three subdivision of the trigeminal nerve, such as Szentágothai and Kiss ('49), Torvik ('56) and Kerr ('63) etc. Physiologically, Kruger et al. ('61) and Kruger and Michel ('62) studied on this problem in the cat, and Nord ('67, '68) and Nord and Kyler ('68) in the rat.

In the study on the nervous system, however, differences of animal species must be enough considered in relation with the animal habit or behavior. Viewed from this point, researches using the rat as the experimental nimal were mainly made by. Torvik. Torvik showed the result by the partial destruction of the semilunar ganglion in accordance with three trigeminal subdivisions, while Clarke and Bowsher ('62) observed on the case by the total section of the trigeminal nerve. But, many important disagreements exist between two results by these authors.

In this paper, the terminations of the primary trigeminal fibers were observed, including the termination to the solitary nucleus via the trigeminal nerve. These results were compared with those by Torvik or Clarke and Bowsher and discussed on the relation with the physiological study by Nord, in addition to the relation with the previous report on the cortical fibers from S I- or S II-face area in the rat (Matano et al., '72). 


\section{Material and Method}

Twenty eight rats (Sprague-Dawley) weighing about 250 gr. were used as the experimental animal. The surgical procedure was done under the nembuthal anesthesia.

The first group (16 cases) was used to study the intraganglionic distribution of the trigeminal subdivision. In 4 cases, the mandibular nerve was sectioned at the exist of the oval foramen. In 8 cases, the ophthalmic or maxillar nerve was severed by the extirpation of the orbit or the destruction of the orbital floor respectively. Furthermore, the inferior alveolar nerve was sectioned in 2 cases and also the lingual nerve in 2 cases.

In the second group of the rat, total ganglionectomy was done in 2 cases. After incision of the infratemporal fossa, the oval foramen was exposed and the bone surrounding this foramen was removed. By this procedure, the semilunar ganglion could be easily exposed and excised without the destruction of the brain. The partial destruction of the ganglion according with the mandibular, maxillar and the ophthalmic subdivisions, was done by the same procedure in 10 cases.

After 2 weeks of the postoperative survivals, the first group (16 cases) of the rat was perfused with $10 \%$ formalin and the semilunar ganglions on both sides were obtained. The post-fixation was done in the same fluid about 5 days. Serial horizontal sections were cut at $10 \mu$ and stained by Gallocyanin or Cresyl violet. Two cases of the total ganglionectomy and 7 cases of the partial one in the second group were prepared by Fink-Heimer method ('67). Between 7-10 days after the operation, the animals were perfused with $10 \%$ neutral formalin and the brain stems obtained were fixed in the same fluid during about 4 weeks. Serial frontal frozen sections were cut at $30 \mu$ and stained by the second procedure of Fink-Heimer method in each interval of $300 \mu$. Three cases of the partial ganglionectomy were fixed in Müller's fixative after 2 weeks of the operation and stained by usual Marchi's method. Kluver-Barrera's preparation of the rat brainstem was used as the control. The nomenclature of the rat's brainstem was referred to atlas of Torvik ('56) and Meessen and Olszewski ('49).

Concerning the subdivision of the spinal trigeminal nucleus, Torvik divided into three subdivisions, that is, the oral, interpolar and the caudal spinal nuclei. Furthermore, at the level of the oral nucleus, he recognized nucleus dorsomedialis, as well as in the case of the mouse shown by Astr $\phi \mathrm{m}$ ('53). In this paper, however, we divided the spinal trigeminal nucleus into two groups: oral portion 
and caudal portion according to rabbit's atlas by Meessen and Olszewski ('49).

\author{
Abbreviations of figure \\ AL: Nucleus alaris \\ CORA: Anterior horn of the spinal cord \\ CUN : Cuneate nucleus \\ DPYR: Pyramidal decussation \\ FLM: Medial longitudinal fasciculus \\ GRA: Gracile nucleus \\ LAT: Subnucleus lateralis caudalis magnocellularis medullae oblongatae \\ (Meessen and Olszewski) \\ LM: Medial lemniscus \\ OLI : Inferior olive \\ OLS: Superior olive \\ PCI : Inferior cerebellar peduncle \\ PCS: Superior cerebellar peduncle \\ PRH: Nucleus prepositus hypoglossi \\ PYR: Pyramidal tract \\ SOL: Nucleus of the solitary tract \\ VMOT: Motor nucleus of trigeminal nerve \\ VSUP: Superior (Main) sensory nucleus of trigeminal nerve \\ VSPO: Spinal trigeminal nucleus, Oral subdivision \\ VSPC: Spinal trigeminal nucleus, Caudal subdivision \\ $z$ : Substantia zonalis, G: Substantia gelatinosa \\ VMES: Mesencephalic nucleus of trigeminal nerve \\ VI: Abducent nucleus \\ VII: Facial nucleus \\ VIII. I: Inferior vestibular nucleus \\ VIII-L: Lateral vestibular nucleus \\ VIII-M : Medial vestibular nncleus \\ VIII-S: Superior vestibular nucleus \\ XII : Hypoglossal nucleus
}

\title{
Observations
}

I. Intraganglionic subdivision of the trigeminal nerve.

The semilunar ganglion of the rat shows three subdivisions according to the ophthalmic, maxillar and the mandibular nerves (Fig. 3). After section of the ophthalmic and the maxillar nerves, the chromatolytic change in the ganglion cell is recognized in the medial and middle portions of the ganglion respectively. The rest of the ganglion cell, which occupies the lateral portion of the ganglion, shows the chromatolytic change after the mandibular nerve section. The motor component belonging to the mandibular nerve penetrates through the lateral part of the ganglion. Furthermore, the localization pattern of the chromatolytic change was studied in the case 
of the inferior alveolar nerve section or the lingual nerve section. The subdivision could not be definitely recognized between the inferior alveolar nerve area and the lingual nerve one. However, the former seems to exist in the medial part, compared to the latter.

In the mandibular area, two types of ganglion cell are clearly recognized. The one is larger in size and much in number and the finer granular Nissl bodies distribute throughout the cytoplasm (Fig. 6), the other is smaller in size and little in number, and the coarse granular Nissl bodies are observed (Fig. 8). After the lingual nerve was sectioned, both types of cells cause the chromatolytic change, as shown in Figs. 7 and 9.

II. Terminations of the primary afferent trigeminal nerve.

Fig. 1 shows a representative case of the total ganglionectomy of the trigeminal nerve. The degenerated fibers proceed into the brainstem as the primary afferent fibers. Terminations are recognized in the superior trigeminal nucleus and the portion dorsomedial to this nucleus (Fig. 1-1). In the rat, the spinal trigeminal nucleus composes from the oral and the caudal portions (VSPO and VSPC). The degenerating terminals are recognized in this spinal nucleus (Figs. 1-3 7). The degenerating fibers of the most caudal level are found at the level of the second cervical cord. Any differences of the the terminal pattern could not be recognized in three layers of the spinal nucleus: that is, substantia zonalis, substantia gelatinosa and nucleus proprius.

Scattering of the terminations in the lateral reticular formation are limited into a narrow portion neighbouring with the spinal nucleus (Fig. 1-3 6). The degenerating fibers scattering in the medial reticular formation or crossing over the midline could not be found throughout the level of the trigeminal nucleus. Of course, the nucleus on the opposite side does not receive the degenerating fibers from the primary fibers. In the Fig. 1-4, a part of the degenerating fiber bundle distributing into the dorsal part of the spinal trigeminal nucleus proceed mediad into the dorsolateral reticular formation and terminate in the most rostral part of the nucleus of the solitary tract.

Fig. 2 shows the case of lesion in the mandibular subdivision of the semilunar ganglion. In general, terminal area occupies the dorsal third of the spinal nucleus, as well as the superior sensory nucleus. However, in some levels, the degenerating terminal area extends into the middle part of the nucleus. In the reticular formation, also, the terminal area is recognized in the dorsal third, compared with the 

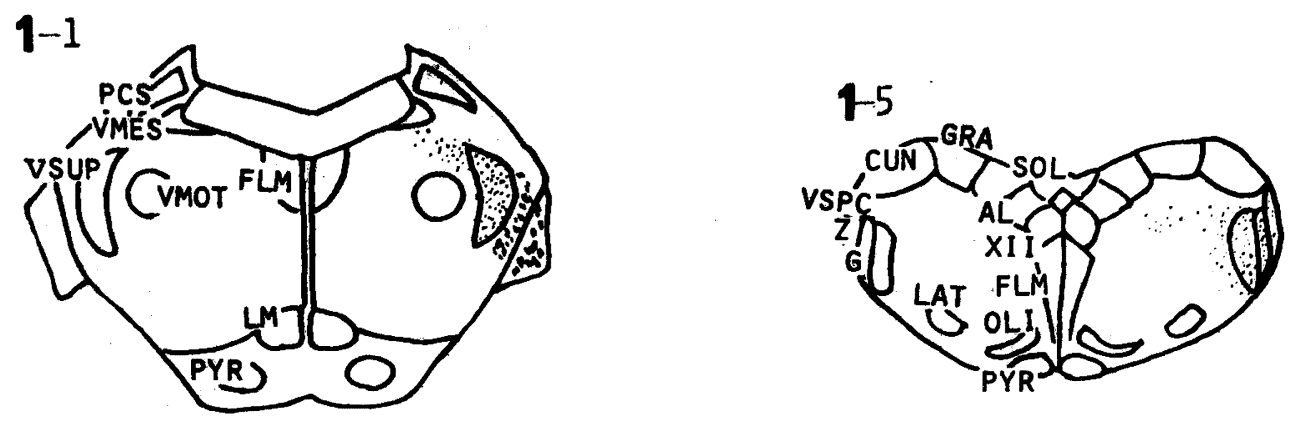

$1-2$

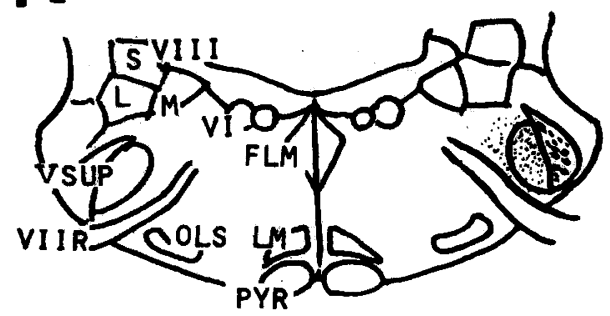

1-6

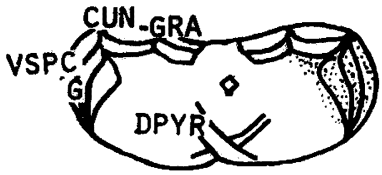

$1-3$

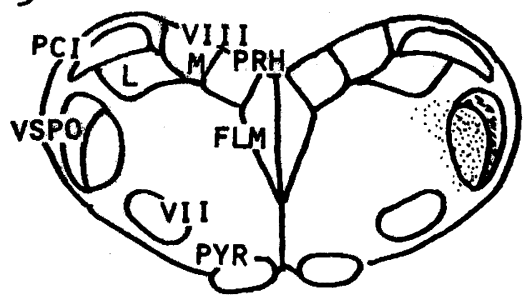

$1-7$
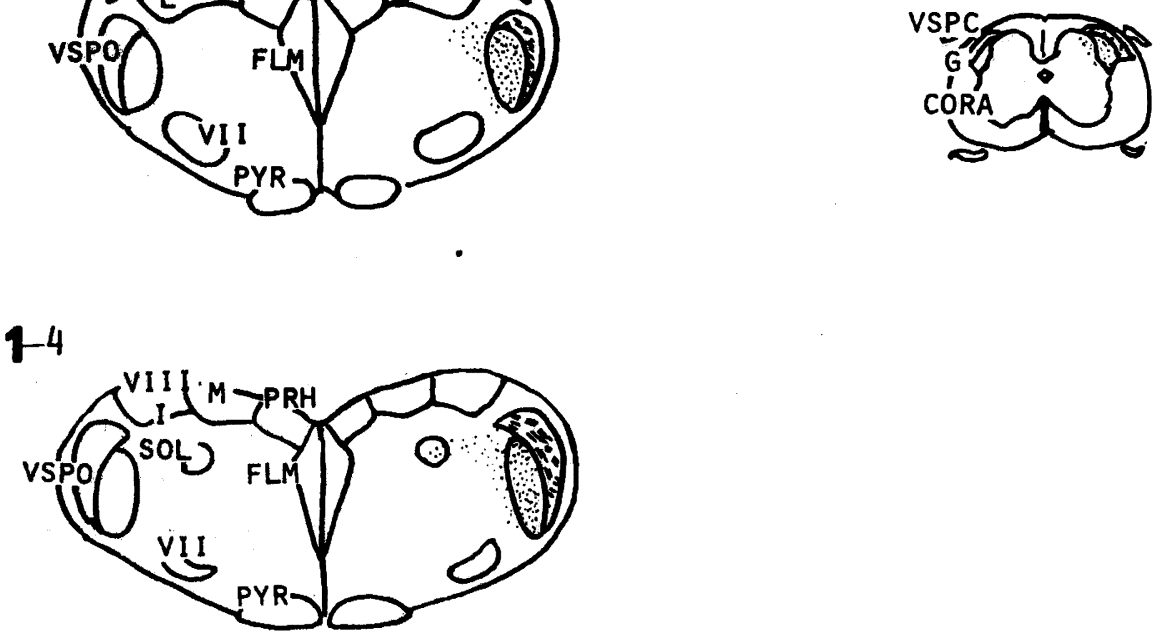

Fig. 1. This figure shows the degenerating terminations of the trigeminal primary afferent nerve in the case of the total excision of the semilunar ganglion. Black spot shows the presynaptic terminals of degenerating axon. 
case of Fig. 1.

In the solitary nucleus at the most rostral level, the degenerated axonal fragments are also recognized, as observed by the case of Fig. 1 (Fig. 2-4, Fig. 5). On the contrary, the case destroyed the opthalmic and the maxillar subdivisions shows no termination of the degenerating axon in the solitary nucleus.

In cases of the partial destruction of the maxillar subdivision, the degenerating fibers terminate in the middle portion of the trigeminal nuclei at any level, and the terminal area in the reticular formation is a narrow part neighbouring with that in the trigeminal nuclei. Moreover, the ventral third of the trigeminal nuclei is filled with the degenerated terminals in cases by the destruction of the ophthalmic subdivision. In the narrow reticular formation neighbouring with this portion, the degenerating fibers also terminate.

\section{Discussion}

Concerning the mandibular subdivision in the semilunar ganglion, Allen ('24) noted that the inferior alveolar nerve cells were much more numerous in the extreme caudal and lateral of the ganglion. In our result, the portion according to the inferior alveolar nerve seems to exist rather medially in the mandibular subdivision of the rat. However, we could not conclude on the definite localization between the inferior alveolar and the lingual nerves as well as the case of the cat observed by Allen.

In general, the primary afferent trigeminal nerve terminated in the nucleus with the dorsoventral direction (Szentágothai, '49, Torvik, '56, Kerr, '63). In our results, the mandibular subdivision was the most dorsal, the maxillar one was the middle and the ophthalmic was the most ventral portion in the trigeminal nuclei, although the overlapping somewhat existed between neighbouring subdivisions. Furthermore, our result in the rat showed that the mandibular or the maxillar area was remarkably larger compared with the ophthalmic one. This fact is considered to show the species specific in rodent.

At any level of the trigeminal nuclei, we could find no termination extending into the medial reticular formation, nor terminals crossing over the midline, which were observed by Torvik or Clarke and Bowsher in the same animal. Of course, degenerated fibers proceeding into the spinal trigeminal nucleus of the opposite side could not be recognized, in contrast to the result by Clarke and Bowsher. Terminations in the reticular formation could be only recognized in the narrow portion neighbouring with the trigeminal nuclei. And also, the same localization pattern of terminals was observed as that 

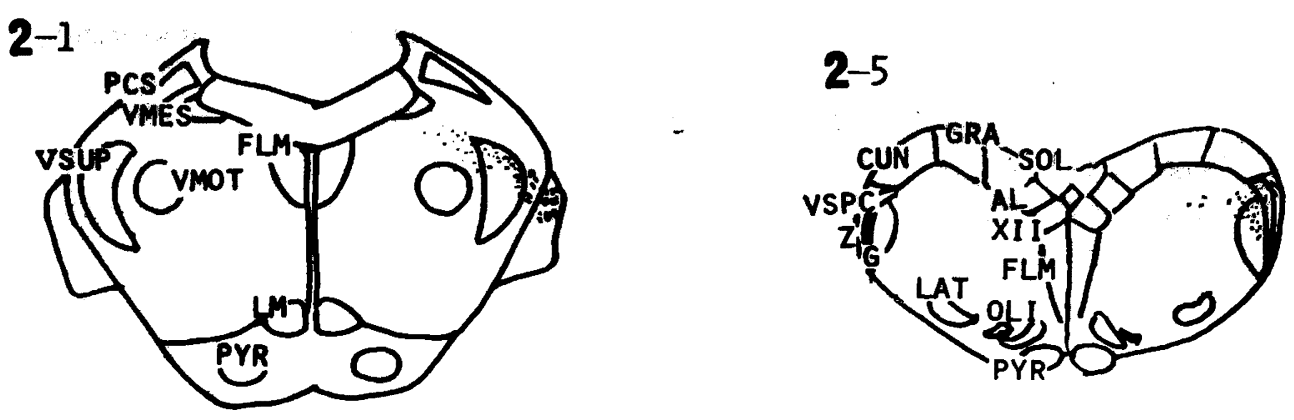

2-2

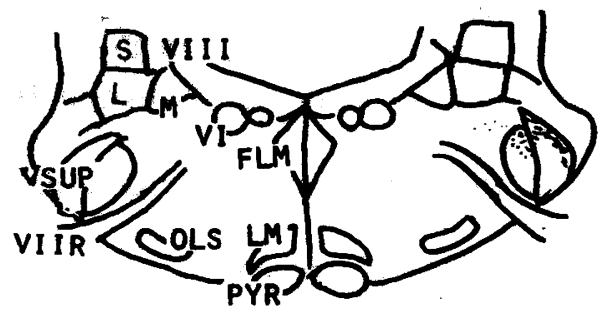

2-6

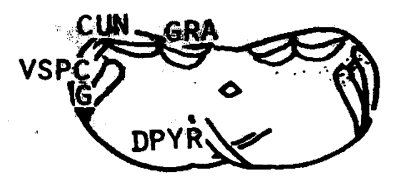

\section{2-3}

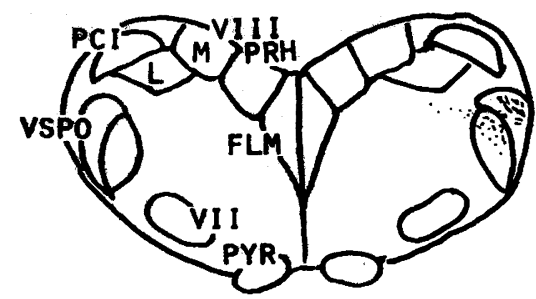

2-7
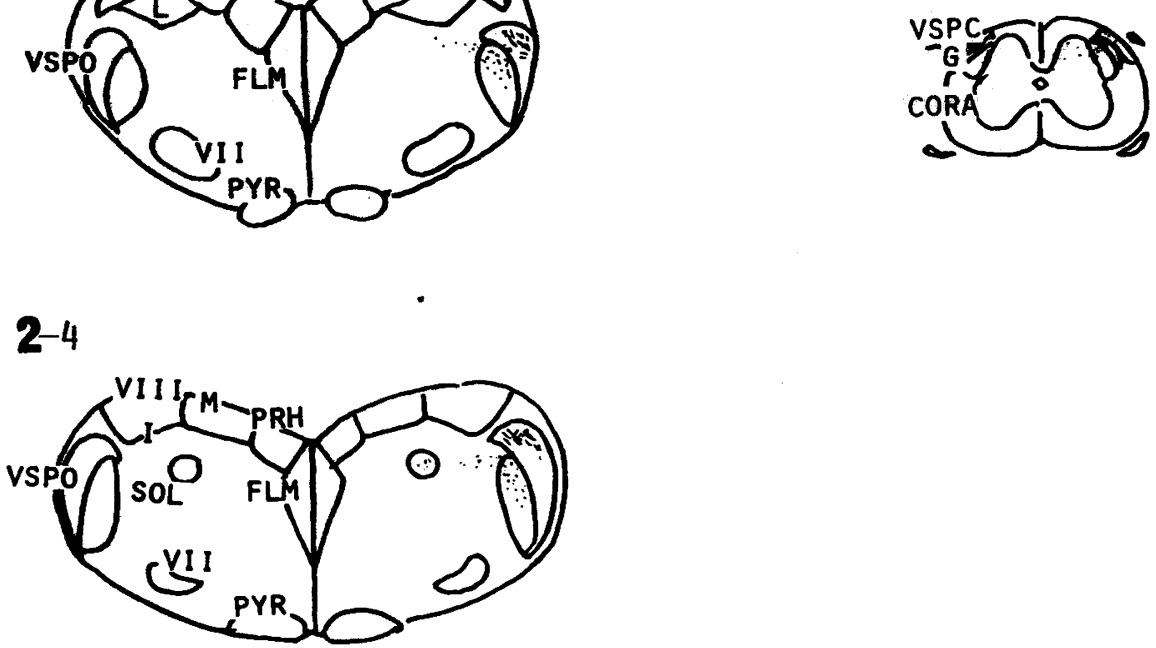

Fig. 2. This figure shows the degenerating terminations of the trigeminal primary afferent nerve in the case of excision of the mandibular subdivision of the semilunar ganglion. Black spot shows the same one as in Fig. 1. 
in the trigeminal nuclei. Our result generally showed the good agreement with the somatotopical map by the physiological study (Nord, '67, '68, Nord and Kyler, '68).

On the other hand, we reported on the terminations to the trigeminal nuclei from the somatic sensory face area (S I, S II) in the rat (Matano et al, '72). The cortical fibers from S I terminated into each sensory trigeminal nucleus, but their terminal pattern did not show any significant localization into the nuclei. On the contrary, the primary fibers terminating in the nuclei showed a localization pattern with the dorsoventral direction in accordance with three subdivisions of the trigeminal nerve. Concerning this problem, further studies on the structural organization in the trigeminal nuclear complex are needful. The recent physiological result (Dubner and Sessle, '71) or the electron-microscopic one (Kerr, '71) seems to show a possibility of solution to this problem, although it is very insufficient in the present state.

Next, several authors demonstrated that a part of the trigeminal primary nerve terminated to the solitary nucleus (Wallenberg, 1897, man ; Astr $\phi \mathrm{m}$, '53, mouse ; Torvik, '56, rat; Kerr, '61, '63, cat ; Clarke and Bowsher, '62, rat; Kimmel et al. '67, cat ; Joseph et al, '68, amphibia etc.). Our result also showed this connection in the rat. It is interesting that this terminal portion in the solitary nucleus accorded with that of the corticofugal fibers from the sensory face area (S I) by our result ('72).

Furthermore, this fiber bundle in the trigeminal nerve arised only from the mandibular subdivision as observed by Torvik, Kerr and Kimmel et al. However, almost no discussions have been done on the localization of the nerve cells of this bundle. In this point, Cushing ('03) previously showed the following course as the suggested one: Some of the inferior ganglion cells, which extend their dendrites into the lingual ramus of the glossopharyngeal nerve, proceed their axons into the trigeminal nerve, via the tympanic nerve, minor petrosus nerve, otic ganglion and the semilunar ganglion.

According to our result, two types of cells in the mandibular subdivision of the smilunar ganglion were recognized. The one was a typical somatosensory ganglion cell as shown in Fig. 6, the other was smaller in number and included the coarse granular Nissl bodies. The latter type of cells is similar to many cells of the geniculate ganglion (Fig. 8). After the lingual nerve section, this type of cells caused the chromatolytic change as wall as the former type of cells. Thus, we suggest that some of cells of the latter type supply their axons for the solitary nucleus in order to conduct the gustatory impulse from the lingual nerve. 


\section{Summary}

1. The terminations of the primary trigeminal fibers were studied using Fink-Heimer technique in the rat.

2. Primary trigeminal afferents terminated in the superior and spinal trigeminal nuclei. In three layers of the spinal nucleus (the zonal, gelatinous and proper), no difference of the terminal density was recognized.

Terminations to the reticular formation were found in a narrow portion of the lateral reticular formation along the column of the spinal nucleus. At the level of the motor trigeminal nucleus, the presynaptic terminals were recognized in the dorsomedial portion to the superior nucleus. There was no termination in the medial part of the reticular formation, nor terminals in the sensory trigeminal nucleus of the opposite side.

A fiber boundle arised from the mandibular subdivision terminated to the most rostral portion of the solitary nucleus.

3. The topographical organization in the trigeminal nucleus was placed with the dorsoventral direction. The mandibular subdivision occupied the most dorsally, the maxillar occupied the middle and the ophthalmic occupied the most ventrally. The mandibular and maxillar subdivisions took up very large area compared with the ophthalmic one.

The same pattern as the dorsoventral direction in the trigeminal nuclei was found in the terminal area of the reticular formation neighbouring with the trigeminal nuclei.

4. In the rat, the above-mentioned topographical localization of the primary fibers did not clearly correspond to the terminal pattern of the corticofugal fibers from S I and S II face areas reported in the previous paper.

5. It was suggested that cells of a peculiar type in the semilunar ganglion supply their axons to the solitary nucleus via the primary trigeminal nerve in order to conduct the gustatory impulse.

\section{References}

Allen, W.F.: Localization in the ganglion semilunare of the cat. J. Comp. Neur., $38: 1-25,1925$.

Astr $\phi$ m, K.E.: On the central course of afferent fibers in the trigeminal, facial, glossopharyngeal, and vagal nerves and their nuclei in the mouse. Acta Physiol. Scand., 29, Suppl., 106 : 209-320, 1953.

Clarke, W. B. and Bowsher, D.: Terminal distribution of primary afferent trigeminal fibers in the rat. Exp. Neurol., $6: 372-383,1962$.

Cushing, H.: The taste fibers and their independence of the $N$. trigeminus. Johns 
Hopkins Hos. Bull., 14: 71, 1903. Cited from "An introduction to neurology" by Herrick, C. J., W. B. Saunders Comp., 1934, p. 293.

Dubner, R. and Sessle, B. J.: Presynaptic modification of corticofugal fibers participating in a feed back loop between trigeminal brainstem nuclei and sensorimotor cortex. From "Oral-facial sensory and motor mechanisms" edited by Dubner, R. and Kawamura, Y., 299-314, Appleton-Century-Crofts. New York, 1971.

Fink, R. P. and Heimer, L.: Two methods for selective silver impregnations of degenerating axons and their synaptic ending in the central nervous system. Brain Res., 4 : 369-374, 1967.

Joseph, B.S., King, R. B. and Whitlock, D.G.: Central distribution of trigeminal primary afferent fibers in Anuran amphibians. Anat. Rec., 160:719-728, 1968.

Kerr, F.W.L.: Structural relation of the trigeminal spinal tract to upper cervical roots and the solitary nucleus in the cat. Exp. Neurol., 4:134-148, 1961.

- : The divisional organization of afferent fibers of the trigeminal nerve. Brain $86: 721-732,1963$.

- : Electronmicroscopic observations on primary deafferentation of the subnucleus caudalis of the trigeminal nerve. From "Oral-facial sensory and motor mechanisms" edited by Dubner, R. and Kawamura, Y., 159-182, AppletonCentury-Crofts. New York, 1971.

Kimmel, D. L., Lesavoy, M. A. and Yeston, N.S.: The central distribution sensory trigeminal nerve fibers. Anat. Rec., $157: 269-270,1967$.

Kruger, L., Siminoff; R. and Witkovsky, P.: Single neuron analysis of dorsal column nuclei and spinal nucleus of trigeminal in cat. J. Neurophysiol., 24 : 333-349, 1961.

Kruger, L. and Michel, F.: A morphological and somatotopic analysis of single unit activity in the trigeminal sensory complex of the cat. Exp. Neurol., 5: 139156, 1962.

Matano, S., Shigenaga, Y., Hiura, T. and Sakai, A.: The cortico-thalamic and -bulbar projections from the somatic sensory face area in the rat. Okajimas Fol. anat. jap.; $49: 249-270,1972$.

Meessen, H. and Olszewski, J. O.: A cytoarchitectonic atlas of the rhombencephalon of the rabbit. S. Karger, Basel \& New York, 1949.

Nord, S. G.: Somatotopic organization in the spinal trigeminal nucleus, the dorsal column nuclei and related structures in the rat. J. Comp. Neur., 130:343-356, 1967.

Nord, S. G.: Receptor field characteristics of single cells in the rat spinal trigeminal complex. Exp. Neurol., $21: 236-243,1968$.

Nord, S. G. and Kyler, H. J.: A single unit. analysis of trigeminal projections to bulbar reticular nuclei of the rat. J. Comp. Neur., 134: 485-494, 1968.

Szentágothai, J. and Kiss, T. : Projection of dermatomes on the substantia gelatinosa. Arch. Neurol. Psychiat., 62 : 734-744, 1949.

Torvik, A.: Afferent connections to the sensory trigeminal nuclei, the nucleus of the solitary tract and adjacent structures. J. Comp. Neur., $106: 51-141,1956$.

Wallenberg, A.: Das dorsale Gebiet der spinalen Trigeminus-wurzel und seine Beziehung zum solitaren Bündel beim Menschen. Deutsche $\mathrm{Ztschr}$. Nervenheilk., $11: 391-405,1897$. 


\section{PLATES}




\section{Explanation of figures}

Fig. 3. This photograph shows the horizontal view of the semilunar ganglion. I; Ophthalmic subdivision, II : maxillar subdivision, III : mandibular subdivision. Gallocyanin staining. $\times 6.5$

Fig. 4. This photograph shows the course and distribution of the degenerating fibers in the spinal trigeminal nucleus in the case of lesion of the mandibular subdivision of the semilunar ganglion. A few degenerated fibers go into the solitary nucleus. Marchi's preparation. $\times 40$.

Fig. 5. Circle surrounding with dotted line of this photograph shows the degenerating terminal area in the oral part of the spinal trigeminal nucleus and the narrow part of the reticular formation neighbouring with it. Fink-Heimer staining. $\times 400$.

Fig. 6. This ganglion cell is one of the larger type in the area of the lingual nerve of the semilunar ganglion. The normal side. Gallocyanin staining. $\times 1,000$.

Fig. 7. This ganglion cell is the same type as shown in Fig. 6. The typical chromatolytic changes are observed. The operated side. Gallocyanin staining. $\times 1,000$.

Fig. 8. These ganglion cells belong to the smaller type in the area of the fingual nerve of the semilunar ganglion. The normal side. Gallocyanin staining. $\times 1,000$.

Fig. 9. This ganglion cell is the same type as shown in Fig. 8. The typical chromatolytic change is recognized. The operated side. Gallocyanin staining. $\times 1,000$. 
Plete I
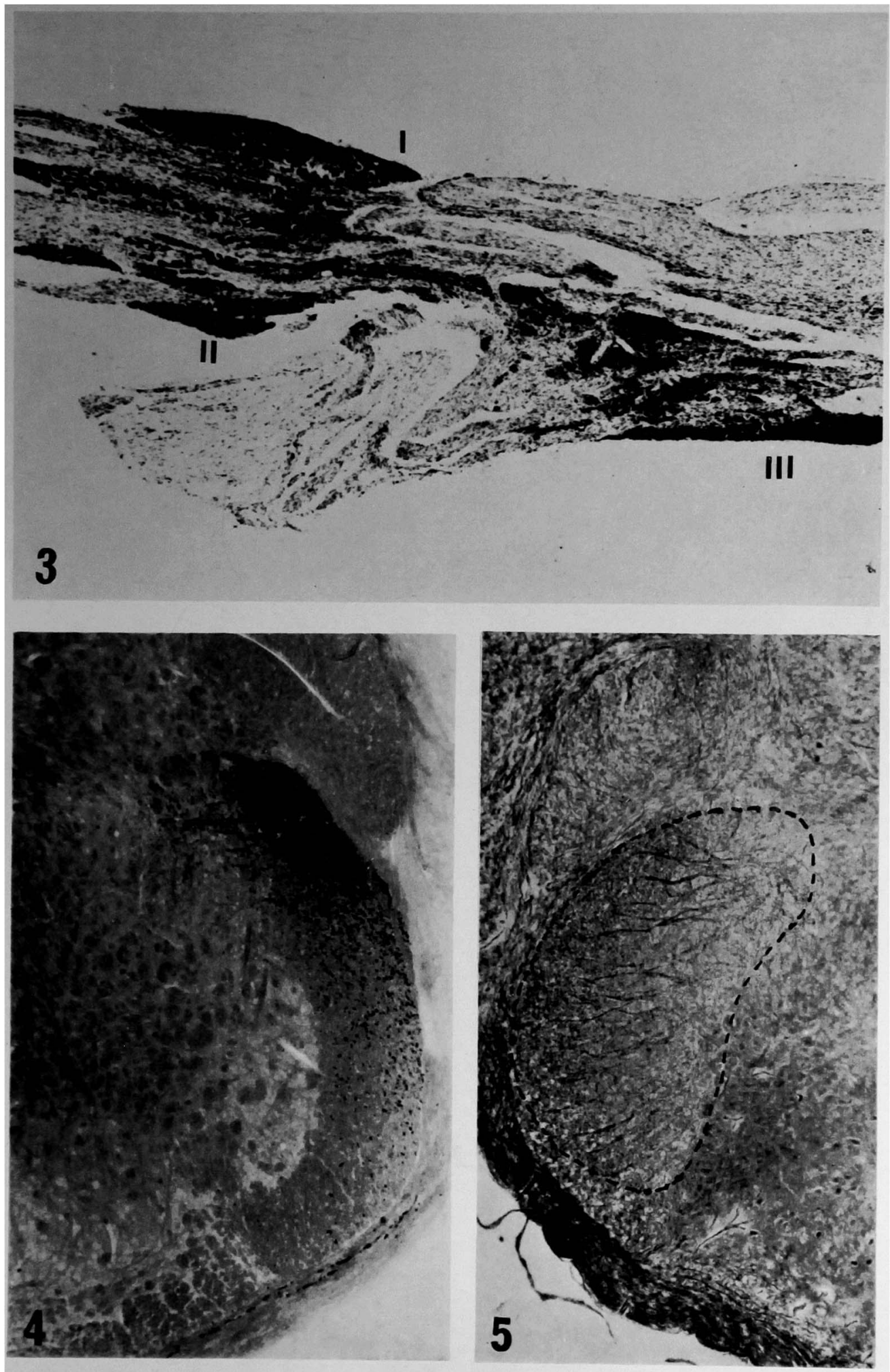

S. Matano et al. 
Plate II

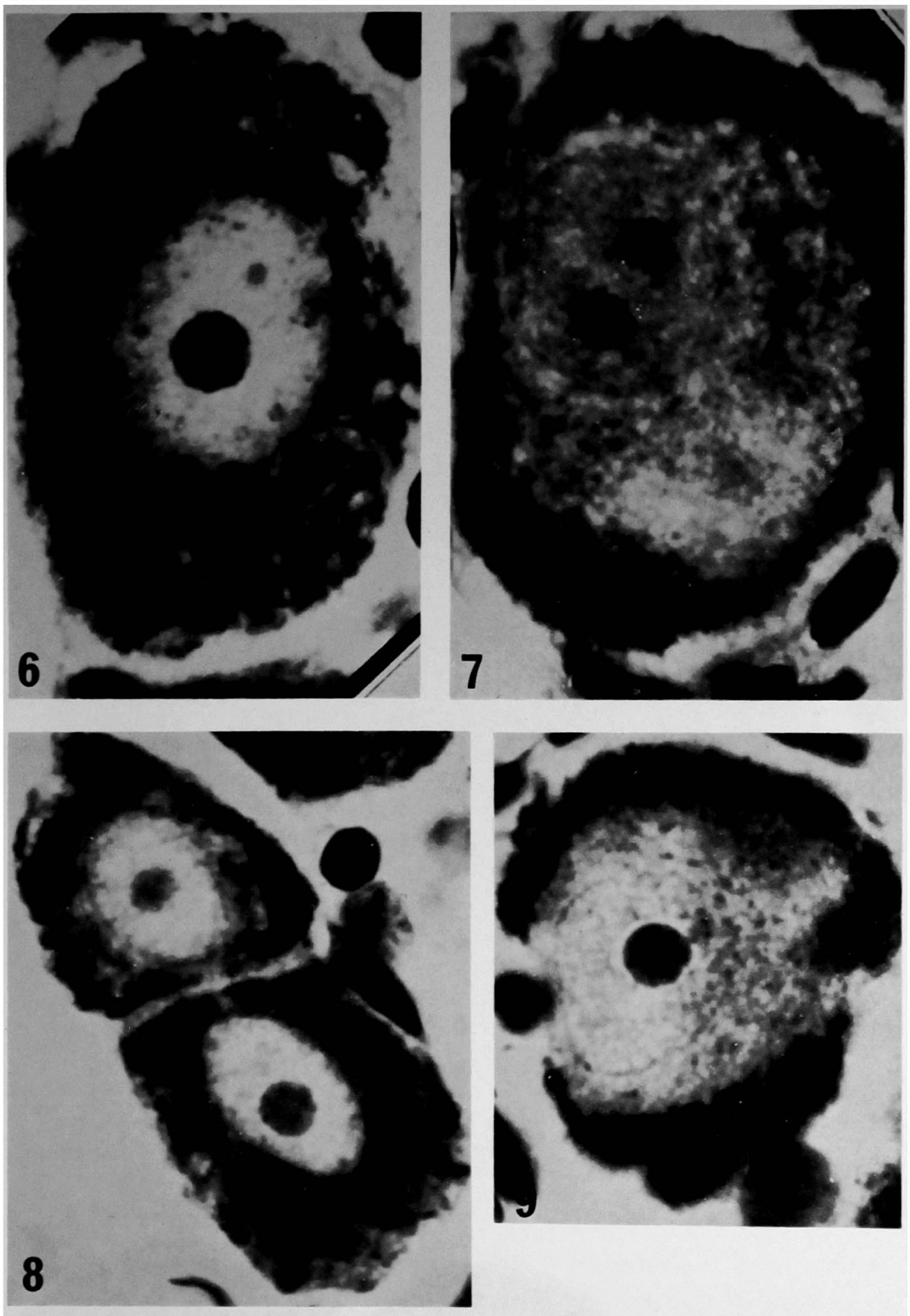

